\title{
MODELING THE SOLAR CYCLE DEPENDENCE OF QUIET-TIME ION UPWELLING AT HIGH GEOMAGNETIC LATITUDES
}

\author{
R. W. Cannata and T. I. Gombosi
}

Space Physics Research Laboratory, The University of Michigan, Ann Arbor

\begin{abstract}
In this letter, we summarize initial results showing how solar cycle variations of the thermosphere modify the composition and intensity of ion upflows at high geomagnetic latitudes. Using an improved version of the one-dimensional hydrodynamic polar wind model developed by Gombosi et al. (1985), we obtain $\mathrm{H}^{+}$and $\mathrm{O}^{+}$vertical flow profiles using different thermospheric states corresponding to solar cycle minimum and maximum. In comparing the quasi-equilibrium flux profiles for both cases, we find that $\mathrm{H}^{+}$ upwelling shows almost no variations in intensity with solar cycle while $\mathrm{O}^{+}$exhibits a 10 -fold increase from solar minimum to solar maximum. These changes result in an $\mathrm{O}^{+}$-dominated upflow at sola maximum while $\mathrm{H}^{+}$flows dominate at solar minimum. We relate these different behaviors to long-term thermospheric changes resulting from solar cycle EUV/UV differences and compare our results with observations.
\end{abstract}

\section{Introduction}

Early theoretical models of high-latitude ion outflows were based on the initial work of Banks and Holzer in 1968 (hereafter referred to as the BH study) in which supersonic outflow of $\mathrm{H}^{+}$and $\mathrm{He}^{+}$ was predicted due to the combined effects of a plasma pressure gradient acting along open geomagnetic field lines and the induced polarization electric field. In considering the gravitational forces acting on the heavier $\mathrm{O}^{+}$and the charge exchange barrier which would limit number densities of upflowing $\mathrm{O}^{+}$in favor of $\mathrm{H}^{+}$ creation, the $\mathrm{BH}$ study concluded that the upflowing $\mathrm{O}^{+}$would be negligibly small compared to $\mathrm{H}^{+}$. Their model predicted thermal $\mathrm{O}^{+}$ fluxes of $10^{5} \mathrm{~cm}^{-2} \mathrm{sec}^{-1}$, about a thousand times less than the $\mathrm{H}^{+}$ outflow. Observations from the Dynamics Explorer-1 satellite (DE 1) later confirmed the predicted supersonic outflow of $\mathrm{H}^{+}$(Nagai et al., 1984) but also identified an unexpected component of suprathermal $(<10 \mathrm{eV})$ heavy ion outflows $\left(\mathrm{O}^{+}, \mathrm{O}^{++}, \mathrm{N}_{2}+\mathrm{NO}^{+}\right)$ over the polar cap. Termed upwelling ion events by Lockwood et al. (1985), these flows were traced back to a spatially-confined region near the dayside polar cap boundary (Waite et al., 1985) and were found to contain a large and sometimes dominant component of $\mathrm{O}^{+}$with fluxes exceeding $10^{8} \mathrm{~cm}^{-2} \mathrm{sec}^{-1}$. As observations accumulated, statistical studies pointed to a strong positive correlation between increases in the solar EUV flux associated with the solar cycle and the occurrence frequency and intensity of upward flowing $\mathrm{O}^{+}$. In sharp contrast, upflowing $\mathrm{H}^{+}$shows no significant solar cycle variations. The study by Yau et al. (1985) indicates a factor of 2-3 increase in 'intense' upflowing oxygen ions as detected by the Energetic Ion Composition Spectrometer (EICS made integrated measurements above a low-energy threshold of $10 \mathrm{eV}$ ) on board DE 1 . Similar solar cycle variations in $\mathrm{H}^{+}$fluxes, however, were not found. This marked solar cycle dependence of $\mathrm{O}^{+}$fluxes, along with the absence of such behavior for $\mathrm{H}^{+}$fluxes, suggests some form of selective thermospheric influence, which is the subject of our present study.

The modulating influence of chemical and collisional processes on ion outflows has been suggested and studied by a number of authors. Banks and Holzer described how gravity, friction and charge exchange would act collectively to impede $\mathrm{O}^{+}$outflows. These effects were modeled using continuity considerations by

Copyright 1989 by the American Geophysical Union.

Paper number $89 \mathrm{GL} 01668$. 0094-8276/89/89GL-01668\$03.00
Moore (1980) and parametrically examined by Barakat et al. (1987). In the Moore (1980) study, parallel or transverse ion acceleration below the level where $n(O)$ equals $n(H)(O / H$ crossover altitude) was required to generate variations in $\mathrm{O}^{+}$upflows. In the Barakat et al. (1987) case, the outflow characteristics were shown to change under different thermospheric and ionospheric conditions when imposed vertical flows for both ion species were applied to simulate a higher altitude energization process. Their modeled $\mathrm{O}^{+}$flows agree well with the observed solar variations mentioned above, however, the limiting $\mathrm{H}^{+}$fluxes they obtained showed a decrease with increasing solar activity. Barakat et al. (1987) point out that this apparent disagreement between modeled and observed $\mathrm{H}^{+}$upwelling behavior may actually be due to differences between energy ranges of the modeled plasma and EICS data. In contrast to these previous treatments, we directly examine the consequences of thermospheric variability on $\mathrm{H}^{+}$and $\mathrm{O}^{+}$upwelling between 200 and $8000 \mathrm{~km}$. The major difference between this study and that of Barakat et al. (1987) is that we impose no upper boundary demand to simulate the removal of $\mathrm{H}^{+}$and $\mathrm{O}^{+}$by unspecified, magnetospheric processes. Instead, we solve the fully-coupled transport equations for ion outflows (c.f. Gombosi and Nagy, 1989), based on a selfconsistent treatment of the significant chemical, collisional and transport processes which are dependent, to a large degree, on the thermospheric temperature and density profiles. Our initial findings reported here, show thermal upwellings of $\mathrm{H}^{+}$and $\mathrm{O}^{+}$which are qualitatively similar to the solar cycle variations seen in the satellite data. Additionally, we show that significantly larger $\mathrm{O}^{+}$flows (exceeding $\mathrm{H}^{+}$flow magnitudes) can exist at solar maximum based solely on thermospheric changes which lead to enhanced $\mathrm{O}^{+}$number densities at constant outflow velocity.

\section{Model Input Parameters}

To determine the extent and influence of thermospheric variability on ion flux character, we compare modeled upflow properties based on a solar minimum and solar maximum thermosphere. This choice simplifies comparisons so that relevant differences can be easily identified. In each case, we adopt thermospheric densities and temperatures derived from the Mass Spectrometer/Incoherent Scatter (MSIS-86) model (Hedin, 1987) and use them to calculate neutralion sources, sinks, and collision frequencies. We generate separate model thermospheres using a daily and averaged $10.7 \mathrm{~cm}$ solar radio flux of $60 \times 10^{-22}$ Watts $\mathrm{m}^{-2} \mathrm{~Hz}^{-1}$ for solar minimum and $180 \times 10^{-22}$ Watts $\mathrm{m}^{-2} \mathrm{~Hz}^{-1}$ for solar maximum. We further assume a flux tube location at $80^{\circ} \mathrm{N} 00^{\circ} \mathrm{W}$ (geomagnetic) and a local solar time of $1200 \mathrm{hrs}$. To limit our comparison to solar cycle (EUV/UV) influences, we consider geomagnetically quiet conditions $(\mathrm{AP}=4)$ during summer (day 183) and use atomic oxygen photoionization frequencies of $1.5 \times 10^{-7} \mathrm{sec}^{-1}$ and $4.5 \times 10^{-7} \mathrm{sec}^{-1}$ to describe solar minimum and maximum conditions, respectively. With these adopted differences, our model can account for the major collisional and chemical processes associated with a solar cycle-dependent thermosphere.

\section{Results and Discussion}

As Moore (1980) pointed out, accidentally resonant charge exchange chemistry closely couples $\mathrm{H}^{+}$and $\mathrm{O}^{+}$flows to the neutral density profiles of atomic oxygen and hydrogen. Therefore, before considering the effects of long-term changes in solar EUV/UV flux on high-latitude ion flow properties, it is useful to examine the 

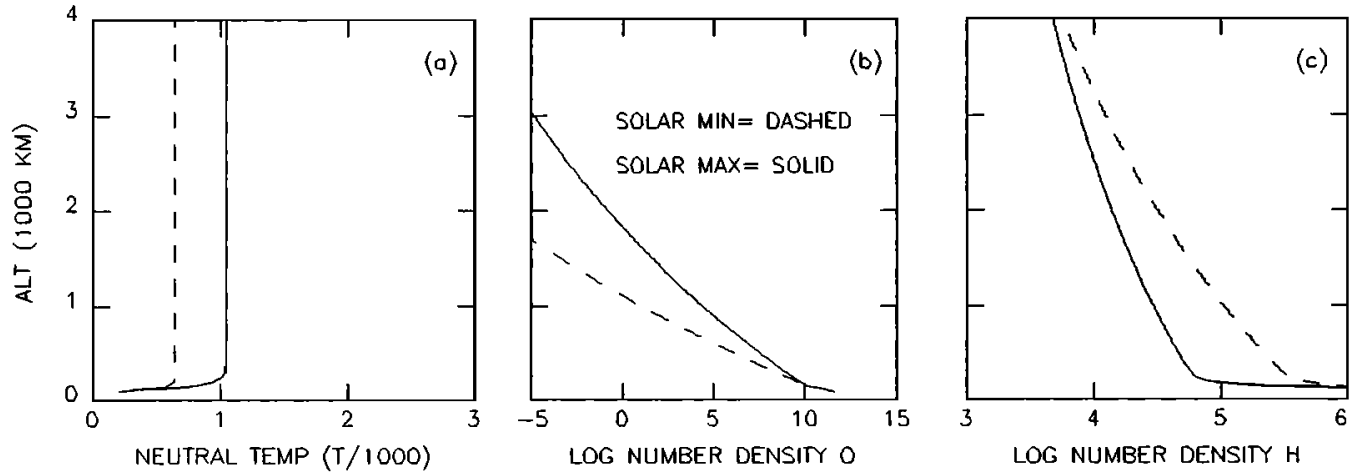

LOG NUMBER DENSITY $H$

Fig. 1. MSIS-86 derived thermospheric profiles for $80^{\circ} \mathrm{N} 00^{\circ} \mathrm{W}$ at $12 \mathrm{LST}$. Profiles are for exospheric temperature (1a), atomic oxygen (1b) and neutral hydrogen number density (1c) for summer solar minimum/maximum conditions. Number densities are $\mathrm{cm}^{-3}$.

differences between the corresponding solar minimum/maximum thermosphere. Molecular as well as atomic thermospheric species are considered in the background gas through which $\mathrm{H}^{+}$and $\mathrm{O}^{+}$, flow therefore, we evaluated changes in all constituents used in our model runs. In the case of molecular nitrogen and oxygen (not shown), increases in local number density were readily apparent between the two cases due to the larger scale heights at solar maximum which are associated with higher exospheric temperatures. The lighter atomic species reacted somewhat differently however. In Figure 1, we compare number density profiles for the major atomic neutral species (hydrogen and oxygen atoms) and the exospheric temperature for the two cases corresponding to solar minimum and solar maximum. Although our MSIS-86 thermosphere profiles extend in altitude to match model calculations up to $8000 \mathrm{~km}$, we only show profiles up to $4000 \mathrm{~km}$ to highlight key structural differences. The significantly (approximately 60\%) greater exospheric temperature associated with enhanced EUV/UV flux during solar maximum, shown in Figure 1a, results in distinct changes in the number density profiles of both major atomic species. In addition to the expected scale height increase in $H$ and $O$ at solar maximum, both constituents are subject to transport which further modifies their profiles. In the case of the heavier
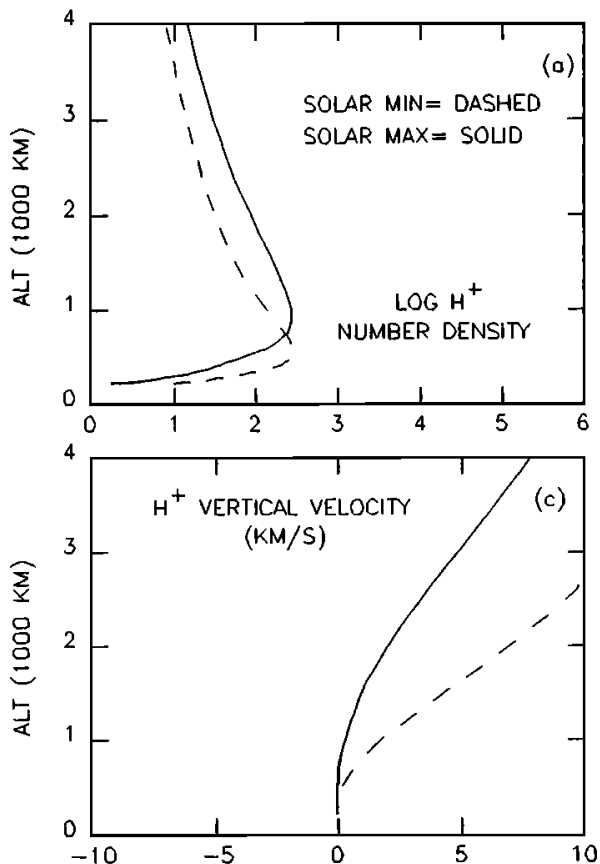

atomic oxygen, local decreases due to transport are small, so the scale height change dominates, resulting in an overall local density enhancement at all altitudes (Fig 1b) at solar maximum. A decrease in $\mathbf{H}$ number densities at solar maximum (Fig 1c) results from enhanced evaporative escape associated with higher thermal velocities. This different behavior of $\mathrm{H}$ and $\mathrm{O}$ at solar minimum /maximum leads to substantial differences for $\mathrm{H}^{+}$and $\mathrm{O}^{+}$upwelling intensities. In fact, the oppositely-directed responses to solar cycle EUV/UV flux of hydrogen and atomic oxygen constitute the most effective thermospheric change in altering the high latitude ion outflow characteristics for the summer conditions examined in this study.

Next, we compare the equilibrium profiles of hydrogen and oxygen ion densities (Fig 2a and $2 \mathrm{~b}$ ) and field-aligned flows (Fig 2c and 2d) for the solar minimum /maximum summer case. Again, we have limited our plots to the $200-4000 \mathrm{~km}$ interval to highlight important differences. In the case of the major ion, $\mathrm{O}^{+}$, enhanced number densities are apparent at all altitudes for solar maximum. This order of magnitude increase in $\mathrm{O}^{+}$results from a combination of two factors. First, higher neutral oxygen scale heights at solar maximum shifts the maximum production level of $\mathrm{O}^{+}$to higher altitudes where loss by reaction with $\mathrm{O}_{2}$ or $\mathrm{N}_{2}$ is less frequent.
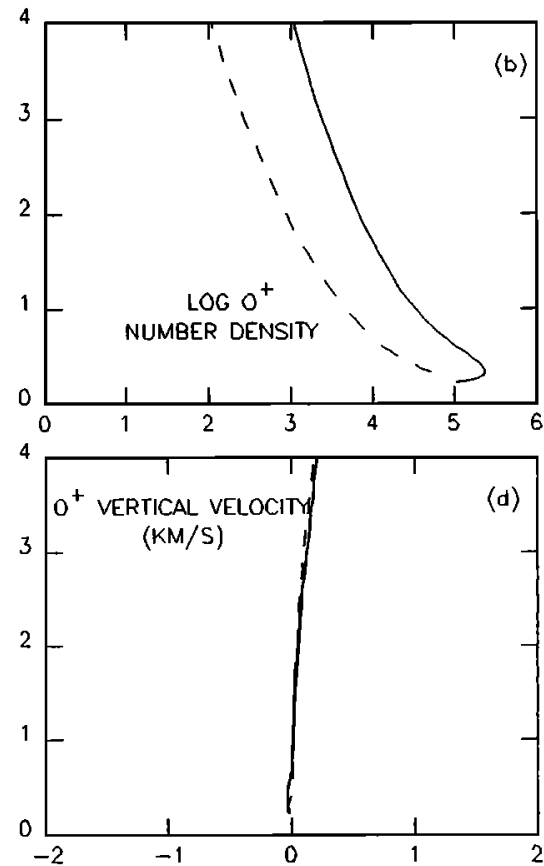

Fig. 2. Quasi-equilibrium profiles of hydrogen and oxygen ion number densities (a, b) and field-aligned flows (c, d) for solar minimum/maximum conditions. 
Secondly, enhanced photoionization rates at solar maximum result in greater $\mathrm{O}^{+}$production. Together, the reduced loss and enhanced production of $\mathrm{O}^{+}$substaintally increase the $\mathrm{O}^{+}$number density at all altitudes during high solar activity. In contrast to this behavior in number density, the field-aligned flow velocities of $\mathrm{O}^{+}$, shown in Figure 2d, exhibit almost no solar cycle variation. For this heavier ion, the diffusive flow which develops from the imposed pressure differential along our model flux tube is constrained to approximately constant velocity by the near balance between gravity, friction and the induced polarization electric field.

For $\mathrm{H}^{+}$, the density and flow velocity profiles shown in Figure $2 \mathrm{a}$ and $2 c$ exhibit a more complex behavior. Since generation of $\mathrm{H}^{+}$via the forward charge exchange reaction is most important near the $\mathrm{O} / \mathrm{H}$ crossover altitude (Moore, 1980), we find the peak in $\mathrm{H}^{+}$number density shifted upwards as the crossover altitude in our adopted MSIS-86 thermosphere moved from $690 \mathrm{~km}$ (solar minimum) to $1290 \mathrm{~km}$ (solar maximum). This change in crossover altitude has important consequences for the $\mathrm{H}^{+}$outflow velocities shown in Figure 2c. Once created, hydrogen ions move primarily in response to the ambipolar electric field established by the major ion, $\mathrm{O}^{+}$. A lower crossover altitude, corresponding to solar minimum, implies a greater vertical distance over which the hydrogen ion can be accelerated by the electric field free from collisions with atomic oxygen. Additionally, since collisions are less frequent above the crossover altitude, hydrogen ions experience less drag and therefore less momentum loss compared to the solar maximum case. As a result, hydrogen outflow velocities are greater at solar minimum (by a factor of 3 at $1000 \mathrm{~km}$ ). In effect, the upward shift in the collision-dominated regime for the solar maximum thermosphere tends to reduce the effectiveness of $\mathrm{H}^{+}$acceleration leading to lower $\mathrm{H}^{+}$outflow velocities at solar maximum.

The distinct variations in density and field-aligned velocities, described above for each ion species, leads to substantial differences in equilibrium fluxes for both solar cycle cases. Figure 3 shows equilibrium flux profiles of $\mathrm{H}^{+}$and $\mathrm{O}^{+}$for solar minimum and maximum. Flux profiles have been normalized to the $1000 \mathrm{~km}$ value to account for the radially-divergent flux tube geometry assumed in our model. The $\mathrm{H}^{+}$flux profile shows almost no dependence on solar cycle conditions reaching an asymptotic value near $3 \times 10^{7} \mathrm{~cm}^{-2} \mathrm{sec}^{-1}$ at $4000 \mathrm{~km}$ for both solar minimum and solar maximum. In contrast to the hydrogen ion, the $\mathrm{O}^{+}$flux profile shows an order of magnitude variation for solar cycle extremes with fluxes increasing from near $6 \times 10^{6} \mathrm{~cm}^{-2} \mathrm{sec}^{-1}$ to approximately $6 \times 10^{7} \mathrm{~cm}^{-2} \mathrm{sec}^{-1}$ at solar maximum. The near constant $\mathrm{H}^{+}$flux profile can be explained in terms of the opposing responses in $\mathrm{H}^{+}$ number density and outflow velocities for solar minimum/maximum periods. Above $4000 \mathrm{~km}$, variations are small and in opposite senses. Slower field-aligned velocities at solar maximum reduce the fluxes between the $\mathrm{O} / \mathrm{H}$ crossover altitude and $4000 \mathrm{~km}$. Below $1000 \mathrm{~km}$, both number density and flow velocity are slightly reduced (compared to solar minimum values) and thus act together to reduce $\mathrm{H}^{+}$fluxes. The $\mathrm{O}^{+}$flux also responds to thermospheric changes but in an entirely different manner. In the lowest altitude region, the peak in $\mathrm{O}^{+}$production shifts upward for solar maximum so that a small downward-directed motion of the oxygen ion away from the source tends to hide the flux profile nose visible in the solar minimum case. Just above the peak production region, substantial fluxes of $\mathrm{O}^{+}$move upward and contribute to the generation of $\mathrm{H}^{+}$ flux via charge exchange with neutral hydrogen. Between the two solar cycle cases, we note the $\mathrm{O}^{+}$flux profile increases by one order of magnitude during solar maximum. By comparison with Figure 2 , we attribute this behavior primarily to the almost 10 -fold increase in $\mathrm{O}^{+}$number density which is directly related to thermospheric compositional changes associated with EUV/UV variations.

Compared with the previous predictions of $\mathrm{BH}$, our field-aligned $\mathrm{O}^{+}$fluxes are significantly greater. We obtain $\mathrm{O}^{+}$flux magnitudes near $6 \times 10^{7} \mathrm{~cm}^{-2} \mathrm{sec}^{-1}$ for solar maximum which are higher than corresponding $\mathrm{H}^{+}$fluxes by a factor of 2 . By comparing Figures $2 \mathrm{~b}$ and $2 \mathrm{~d}$, we note the larger fluxes are a consequence of increased $\mathrm{O}^{+}$ concentrations and not the result of higher diffusion velocities. Since our fluxes are 100 times greater than that predicted by $\mathrm{BH}$, we briefly examined model assumptions and found, (1) thermospheric $H$ densities for the three different neutral atmosphere cases assumed by BH were larger by a factor of 2 to 10 compared to MSIS-86 cases for corresponding exospheric temperatures and (2), the $3000 \mathrm{~K}$ electron temperature $\left(T_{e}\right)$ assumed by $B H$ was significantly lower than the $7500 \mathrm{~K}$ temperature we calculate from a specified downward heat flux. Our calculated, low altitude profile of $T_{e}$ is consistent with DE 2 observations of low altitude, cusp region electron temperatures reported by Curtis et al.(1985). Since a hydrogen-rich atmosphere and lower $\mathrm{T}_{e}$ could impede $\mathrm{O}^{+}$flows through friction and a reduced ambipolar electric field, we decided to examine the effects of the BH assumptions on our model.

We examined the effects of high neutral $H$ densities and low $T_{e}$ through suitable modifications of our model for three cases. In the first case, we artificially enhanced the neutral $\mathrm{H}$ density by a factor of 10 . For the second case, we lowered the downward electron flux by one order of magnitude to $5 \times 10^{-4} \mathrm{erg} \mathrm{cm}^{-2} \mathrm{sec}^{-1}$ which reduced the $T_{e}$ to $3800 \mathrm{~K}$. In the third case, we combined both modifications to match the $\mathrm{BH}$ assumptions. We summarize our results in Table 1. For the case of enhanced neutral $\mathbf{H}$ density (column labeled "enhanced $\left.\mathrm{H}^{\prime}\right), \mathrm{H}^{+}$upflows increased by a factor of 8 with only negligible effect on $\mathrm{O}^{+}$flows. For the second case, decreases in $\mathrm{T}_{\mathrm{e}}$ (column labeled " $\mathrm{T}_{\mathrm{e}}=3800 \mathrm{~K}$ ") reduced the $\mathrm{O}^{+}$flux by a factor of 10 with only a small (factor of 2 ) decrease in the $\mathrm{H}^{+}$flux. The decrease in $\mathrm{O}^{+}$flux with lowered $\mathrm{T}_{e}$ is qualitatively similar to results obtained by Barakat and Schunk (1983). In their study, $\mathrm{T}_{\mathrm{e}}$ was found to be the most important parameter with regard to $\mathrm{O}^{+}$polar wind solutions. When both effects were considered (column labeled "combined effects"), $\mathrm{O}^{+}$fluxes decreased by a factor of 80 while $\mathrm{H}^{+}$ flows increased by a factor of three. For this latter case, we obtain the same flux magnitudes for both ion species as BH when we use their assumptions in our model. Based on these findings, we conclude that the differences between our model results and those of
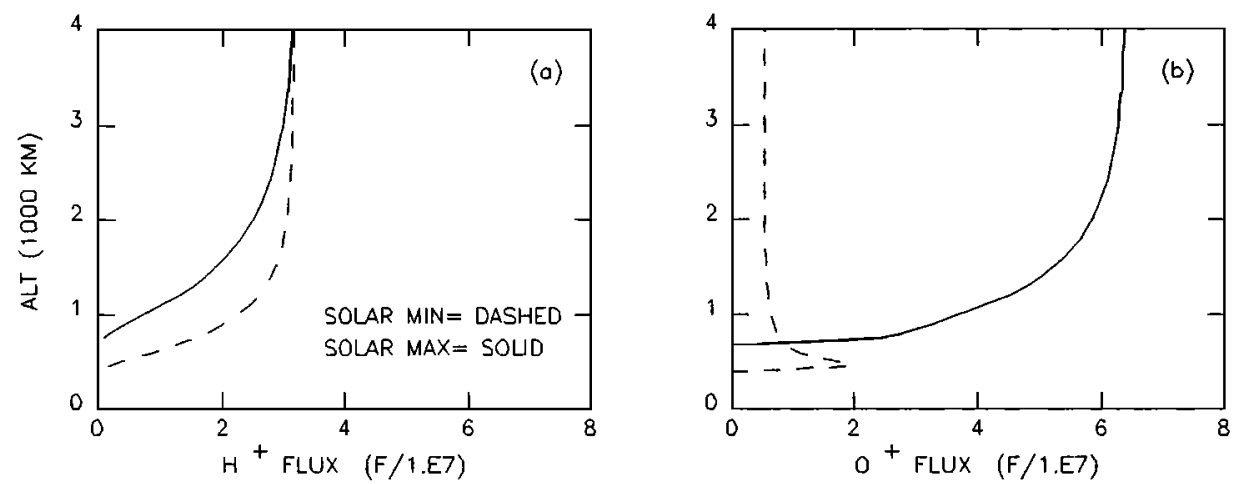

Fig. 3. $\mathrm{H}^{+}$(a) and $\mathrm{O}^{+}$(b) flux profiles (ions $\mathrm{cm}^{-2} \mathrm{sec}^{-1}$ ) normalized to an altitude of $1000 \mathrm{~km}$ for summer solar minimum/maximum conditions. 
TABLE 1. Equilibrium fluxes (ions $\mathrm{cm}^{-2} \mathrm{sec}^{-1}$ ) for summertime solar maximum conditions for test cases using the Gombosi polar wind model with low electron temperature, high neutral hydrogen density or a combination of both assumptions. Fluxes listed under the "combined effects" column are similar in magnitude to those predicted by Banks and Holzer (1968).

\begin{tabular}{lllll}
\hline & $\begin{array}{l}\text { unmodified } \\
\text { model }\end{array}$ & enhanced $\mathrm{H}$ & $\mathrm{T}_{\mathrm{c}}=3800 \mathrm{~K}$ & $\begin{array}{l}\text { combined } \\
\text { effects }\end{array}$ \\
\hline $\mathrm{H}^{+}$ & $3.3 \times 10^{7}$ & $2.8 \times 10^{8}$ & $1.3 \times 10^{7}$ & $1.2 \times 10^{8}$ \\
$\mathrm{O}^{+}$ & $6.5 \times 10^{7}$ & $9.0 \times 10^{7}$ & $7.2 \times 10^{5}$ & $8.1 \times 10^{5}$ \\
\hline
\end{tabular}

$\mathrm{BH}$ (we obtain 100 times more $\mathrm{O}^{+}$flux) arise from the more realistic MSIS hydrogen densities and electron temperatures used in our model.

\section{Comparison with Observations}

A quantitative comparison between the flux magnitudes of the thermal $(<\mathrm{eV}$ ) plasma flows described by our model and in-situ, outflow measurements is difficult to make directly since the satellite data are measured over an integral energy range above $10 \mathrm{eV}$. Furthermore, the fluxes we obtain do not necessarily represent the total ion outflow measured at higher satellite altitudes since further energization processes can occur which will alter the phase-space distributions of particles. In fact, Moore et al. (1986) found upwelling fluxes on the order of $10^{9} \mathrm{~cm}^{-2} \mathrm{sec}^{-1}$ and, at times, $10^{10}$ $\mathrm{cm}^{-2} \mathrm{sec}^{-1}$ (C. J. Pollock, private communication, 1989) in the same region we model suggesting that further energization of the plasma is likely. With these limitations in mind, we make a qualitative comparison and find the solar cycle trends in our modeled fluxes of $\mathrm{H}^{+}$and $\mathrm{O}^{+}$are remarkably similar in character to those seen in direct and indirect outflow measurements. In the latter case, in-situ compositional variations in the near-earth plasma sheet can yield information in the form of ion outflows necessary to populate this region assuming steady state conditions. Young et al. (1982) for example, examined 48 months of ion composition data collected by the ESA/GEOS 1 and 2 satellites at geosynchronous orbits and found a strong correlation ( $R=0.94)$ between monthly averaged $\mathrm{O}^{+}$ densities (0.9-15.9 keV) and the F10.7 solar radio flux (80-220 $\times 10^{-}$ 22 Watts $\mathrm{m}^{-2} \mathrm{~Hz}^{-1}$ range) during quiet geomagnetic conditions. For the same period, the $\mathrm{H}^{+}$densities showed only a weak F10.7 dependence (although some ambiguity exists in the $\mathrm{H}^{+}$densities as the authors point out since solar as well as terrestrial sources contribute to the $\mathrm{H}^{+}$plasma sheet density) implying that $\mathrm{O}^{+}$outflows are much more sensitive to F10.7 (and therefore EUV/UV) variations. Our modeled flux behavior also agrees with the statistical studies of Yau et al. (1985), in which $\mathrm{O}^{+}$but not $\mathrm{H}^{+}$, outflows were higher at solar maximum. These findings along with the indirect evidence presented by Young et al. suggest an outflow modulation in $\mathrm{O}^{+}$and $\mathrm{H}^{+}$fluxes closely tied to solar cycle EUV/UV variations and qualitatively similar to our model results.

\section{Conclusion}

We solved the coupled, time-dependent continuity, momentum and energy equations for a two-ion quasi-neutral plasma along an open flux tube at high geomagnetic latitude. Equilibrium solutions were obtained for different thermospheric states corresponding to summer solar minimum and solar maximum. Equilibrium fluxes in both ion species profiles were compared and analyzed to determine the influence of solar cycle dependent thermospheric variability. We note the following:

1. $\mathrm{O}^{+}$fluxes exhibit a strong EUV/UV dependence resulting in a 10 -fold increase in $\mathrm{O}^{+}$upflow at solar maximum over solar minimum. Fluxes of $\mathrm{O}^{+}$with magnitudes near $10^{8} \mathrm{~cm}^{-2} \mathrm{sec}^{-1}$ are possible at solar max and may, at times, exceed $\mathrm{H}^{+}$fluxes .

2. The $\mathbf{H}^{+}$flux exhibits essentially no upflow variations above $4000 \mathrm{~km}$ under similar conditions. Small, factor of 2-3 changes with solar cycle were noted in $\mathrm{H}^{+}$fluxes but only below about $4000 \mathrm{~km}$.

3. Increases in the $\mathrm{O}^{+}$upflow during solar maximum can be explained by EUV/UV-related thermospheric increases in neutral atomic oxygen density and, to a lesser degree, increases in the photoionization frequency.

4. Roughly equal and oppositely-directed responses to EUV/UV variations for $\mathrm{H}^{+}$number density and field-aligned velocities account for the steady upflow of $\mathrm{H}^{+}$above $4000 \mathrm{~km}$. Below this altitude, reduced $\mathrm{H}^{+}$field-aligned velocity and lower densities act to reduce $\mathrm{H}^{+}$fluxes at solar maximum.

5. Despite the restrictive constraints imposed by our choice of season and geomagnetic activity level, our modeled $\mathrm{O}^{+}$and $\mathrm{H}^{+}$ fluxes display a solar cycle dependence qualitatively similar to satellite observations.

Acknowledgements. The authors are grateful to Dr. A.F. Nagy and Dr. J Kozyra for illuminating discussions. This work was supported by NSF grant ATM-8910704. We also wish to acknowledge the National Center for Atmospheric Research, sponsored by NSF, for the computing time used in this research.

\section{References}

Banks, P. M., and T.E. Holzer, The polar wind, L. Geophys. Res., 73, 6846-6854, 1968.

Barakat, A. R.and R. W. Schunk, $\mathbf{O}^{+}$ions in the polar wind, $\mathbf{J}$ Geophys. Res., 88, 7887-7894, 1983.

Barakat, A. R., R. W. Schunk, T., E., Moore and J., H., Waite Jr., Ion escape fluxes from the terrestrial high-latitude ionosphere, L. Geophys. Res., 92, 12255-12266, 1987.

Curtis, S. A., W. R. Hoegy and L. H. Brace , Cusp altitudinal electron temperature gradient: Dynamics Explorer 2 implications for heating mechanisms, J.Geophys. Res., 90, 4415-4419, 1985.

Gombosi, T. I.and A. F. Nagy, Time-dependent modeling of fieldaligned current-generated ion transients in the polar wind, $L$ Geophys. Res. , 94, 359-369, 1989.

Hedin, A. E., MSIS-86 thermospheric model, L. Geophys. Res., 22, 4649-4662, 1987.

Moore, T. E., Modulation of terrestrial escape flux composition (by low-altitude acceleration and charge exchange chemistry), $\mathbf{L}$ Geophys. Res., 85, 2011-2016, 1980.

Moore, T. E., C. R. Chappell, M. Lockwood, and J. H. Waite, Jr., Suprathermal ion signatures of auroral acceleration processes, $\mathrm{L}$ Geophys. Res., 90, 1611-1618, 1985.

Moore, T. E., M. Lockwood, M. O. Chandler, J. H. Waite, Jr., C. R. Chappell, A. Persoon, and M. Suguira, Upwelling $\mathrm{O}^{+}$ion source characteristics, J.Geophys. Res., 21, 7019-7031, 1986.

Nagai, T., J. H. Waite, Jr., J. L. Green, C. R. Chappell, R. C. Olsen, and R. H. Comfort, First measurements of supersonic polar wind in the polar magnetosphere by DE-1/RIMS, Geophys. Res. Lett., 11, 669-672, 1984.

Yau, A. W., P. H. Beckwith, W. K. Peterson, and E. G. Kelly, Long-term (solar cycle) and seasonal variations of upflowing ionospheric ion events at DE 1 altitudes, J. Geophys. Res., 20 , 6395-6407, 1985.

Young, D. T., H. Balsiger, and J. Geiss, Correlations of magnetospheric ion composition with geomagnetic and solar activity, L. Geophys. Res., 87, 9077-9096, 1982.

R.W. Cannata and T.I. Gombosi, Space Physics Research Laboratory, Department of Atmospheric, Oceanic and Space Sciences, 2455 Hayward, Ann Arbor, Michigan 48109-2143.

(Received July 11, 1989; accepted July 24, 1989.) 